Andrzej Zieliński

Uniwersytet Jagiellonski w Krakowie andrzej.zielinski@uj.edu.pl

\title{
Sobre el origen del valor frecuentativo de $<$ saber + infinitivo $>$
}

\section{Resumen:}

El objeto del presente estudio es ofrecer una nueva propuesta del origen del valor frecuentativo de la perífrasis verbal $<$ saber + infinitivo $>$, diferente del calco semántico del quechua, como propone Vázquez (1980), y del cultismo, como sostiene Lida de Malkiel (1948). Asimismo, se determinará la trayectoria semántica que experimentó el verbo auxiliar.

Palabras clave: perífrasis verbal, patrón de lexicalización, reajuste semántico

\begin{abstract}
:
On the Origin of the Frequentative Meaning of $<$ saber + Infinitive $>$ The main purpose of this paper is to offer a new proposal about the origin of the frequentative meaning of the verbal periphrasis $<$ saber + infinitive $>$, different from Vázquez's (1980) hypothesis of a loan-translation from Quechua, and from Lida de Malkiel's (1948) interpretation of this as a cultism. Furthermore, the semantic trajectory that the auxiliary verb has followed will be traced.
\end{abstract}

Keywords: verbal periphrasis, lexicalization pattern, semantic change 


\section{Introducción}

El objeto del presente estudio es determinar el origen del valor frecuentativo de la perífrasis verbal ${ }^{1}<$ saber + infinitivo $>$, cuya lectura implica que la actividad denotada por el infinitivo ocurre más de una vez, sin que al emisor le interese destacar ni su comienzo ni su final (1).

Si bien en el español del Río de la Plata su empleo desbancó por completo a la construcción $<$ soler + infinitivo $>$ (1a) (Lida de Malkiel, 1948: 269; Zamora, 1974: 427), en la zona andina y en el sur de Colombia (1b-c) - quizás debido al prestigio del español rioplatense-, el valor frecuentativo de $<$ saber + infinitivo $>$ admite incluso lecturas limitadas temporalmente (1d) (RAE-ASALE, 2009: § 28.4h). Se distingue así de $<$ soler + infinitivo $>$, que — como bien se sabe - carece de la forma verbal perfectiva (*solió, *ha solido, etc.).

(1) a. El vulgo no confunde jamás las condiciones de verdadero, bueno y bello; siempre sabe distinguir (Bunge, Principios de psicología individual y social, 1928, Argentina, CORDE);

b. [los indios] muchos estan Ricos mas bien tratados y mantenidos que en sus tierras como se an abitado al trabajo saben ganar de comer (Anónimo, Carta de Damián de Lavandera, 1481, Bolivia, CORDIAM);

c. McCarry no es un escritor de los que suelen denominarse „electrizantes", pero en su tono gris, asordinado, sabe ir pegando los hechos de tal manera que el lector no enloquece de afecto (Revista Hoy 08-14/11/1978, Chile, CREA);

d. Ella supo venir a casa, de visita una o dos veces por mes (RAE-ASALE, 2009: § 28.4h).

No abunda la bibliografía sobre el tema, por lo que revisaremos los dos planteamientos más conocidos: el de Lida de Malkiel (1948)

${ }^{1}$ Por perífrasis verbal se entiende una unión semántico-sintáctica de, al menos, dos verbos: uno en forma personal, llamado verbo auxiliar, y otro en forma no personal, denominado auxiliado. El resultado constituye una unidad cabal de carácter conceptual, no siempre predecible a través de los elementos que la componen (Olbertz, 1998; Zieliński, 2014). 
- mayoritariamente aceptado por los investigadores que se ocupan de perífrasis verbales (Yllera, 1980; Olbertz, 1998; Fernández de Castro, 1999; García Fernández, 2006) — , y el de Vázquez (1980), contrastándolos con los datos a los que los filólogos de hoy tenemos acceso gracias a los avances, por una parte, de la lingüística de corpus y, por otra, de los estudios sobre conceptualización que ofrece la gramática cognitiva. Creemos que es fundamental llevar a cabo un serio estudio histórico para determinar el origen de esta perífrasis y observar los cambios que ha sufrido el verbo saber en ella.

Nuestro trabajo se divide en dos partes. En primer lugar $(\S 1)$, tras examinar con mirada crítica los dos principales planteamientos sobre su procedencia, nos ocuparemos del origen del valor frecuentativo de la construcción perifrástica $<$ saber + infinitivo $>$ y plantearemos una nueva hipótesis tomando como base el patrón de lexicalización de Talmy (1985). En segundo lugar (§2), basándonos en la hipótesis propuesta, presentaremos la evolución semántica que experimenta el verbo auxiliar saber hasta adquirir el significado de frecuentatividad. Cierran este estudio las conclusiones a las que hemos llegado (§ 3).

\section{Origen}

Hasta el momento únicamente disponemos de dos hipótesis - muy diferentes - sobre el origen del valor frecuentativo de saber: por un lado, se asocia con el influjo de una lengua indígena de América y, por otro, con una tradición culta que se remonta al mundo grecolatino.

Dado que se observa (Kany, 1994: 249) la persistencia del valor frecuentativo en zonas de Sudamérica (Argentina, Paraguay, Bolivia, Perú, Chile y sur de Colombia), Vázquez (1980) cree que el origen de la perífrasis se relaciona con el dominio lingüístico del quechua, cuyo verbo yachana codifica tanto el conocimiento adquirido como la idea frecuentativa. Aunque esta hipótesis explica que el influjo quechua contribuye al mantenimiento del valor en cuestión en algunas variedades del español americano, la lectura frecuentativa de la perífrasis se documenta en la lengua medieval peninsular, como demuestra el Libro de Alexandre (2a-b), fechado a mediados del siglo XIII. 
(2) a. esta faz las iglesias sagradas vïolar/ sabe a los perlados de mesura sacar (LAlex. vv. $2368 \mathrm{c}-\mathrm{d}$ );

b. omne que tantos sabe judiçios delivrar/ por qual juïçio dio, por tal debe passar (LAlex, vv. $2330 \mathrm{c}-\mathrm{d}$ ).

Mucho antes que Vázquez, Lida de Malkiel (1948), con criterio diacrónico, destacó el origen culto del valor frecuentativo de $<$ saber + infinitivo $>$, reflejo de una larga tradición que, en su opinión, remite a la cultura grecolatina y pasó a las lenguas románicas gracias, sobre todo, a la repercusión de la Vulgata, texto en el que la construcción, a juicio de esta prestigiosa investigadora, está ampliamente documentada. Sin embargo, ni Lewis y Short (1879/1969: s.vv. scio, nescio y sapio) ni Ernout y Meillet (1951: s.vv. scio, nescio y sapio) reconocen su empleo frecuentativo en ningún tipo de latín. Es más: los pasajes latinos proporcionados por Lida de Malkiel resultan sumamente dudosos (3a), ya que no se puede descartar la lectura con el significado de 'habilidad', como tampoco en la ampliación del estudio que hemos realizado consultando Brepolis. Como mucho podemos considerarlos como ejemplos ambiguos (en un contexto puente), a partir de los cuales pudo surgir la idea aspectual. Así, en (3b) no se sabe si los cerdos salvajes, sujeto omitido en el enunciado, poseen la habilidad de confundir sus huellas o bien ya suelen hacerlo, dado que lo habían aprendido con anterioridad.

(3) a. Stare loco nescit, micat auribus et tremit artus

(Virgilio, Geórgicas, III, 84 apud Lida de Malkiel 1948: 273);

b. feri sapiunt vestigia palude confundere, urina fugam levare (Plinio, Historia natural VIII, 208-209, BREPOLIS);

[(los cerdos salvajes) saben/suelen confundir las huellas en la ciénaga, aligerar la huida con la orina].

Tampoco la búsqueda que hemos realizado en biblias medievales ha dado resultados positivos. Obsérvense las traducciones medievales del mismo pasaje de la Vulgata (4b-d), cuya lectura - siempre en contexto negativo, lo que favorece el solapamiento con la frecuentatividad (vid. infra) - no deja lugar a dudas y apunta, evidentemente, al significado prototípico de la construcción. 
(4) a. labor stultorum adfliget eos qui nesciunt in urbem pergere (Vulgata);

b. So trabaio quexara a los locos que non saben andar por la uilla (Cod. E6);

c. la cuyta a los locos los faze afanar por non saber yr ala çibdat (Cod. EV);

d. El trabaio de los nesçios lo cansara el qual non sabe yr a çibda (E4); (Eclesiastés 10, 15 apud Enrique-Arias, 2008).

Esto nos lleva a pensar que el origen aspectual de la construcción no se debe a influjo quechua, como defiende Vázquez (1980), ni tiene raíces cultas, como sostiene Lida de Malkiel (1948), sino que, como intentaremos probar a continuación, puede presentar un carácter popular, relacionado con la conceptualización de la repetición de una actividad como mera rutina de la vida cotidiana. Es lo que demuestra la serie formada por las cuatro construcciones frecuentativas del castellano medieval (5) (Yllera, 1980: 199-204, Zieliński, 2010: 676-678), que presentan el mismo patrón de lexicalización (lexicalization pattern), en términos de Talmy (1985), a través del cual se codifican con más facilidad los elementos cultural y cognitivamente fundamentales para una comunidad lingüística.

En primer lugar, nótese que lo que tienen en común <soler $(<$ SŎLERE 'tener costumbre', y este, a su vez, procede de SŎLUM 'suelo, lugar donde uno se asienta más frecuentemente') + infinitivo $>$ ( $c f$. fr. $s$ 'habituer 'acostumbrarse', derivado de HABITUS), <usar de + infinitivo $>$ ( $c f$. ing. use to), $<$ saber + infinitivo $>$ y $<(a)$ costumbrar + infinitivo $>$ es que el valor que han adquirido deriva de la acepción léxica que indica la rutina en la realización de eventos o de actividades diarias (Zieliński, 2010: 676-678):

(5) a. E non só yo la tu asna en que tú sueles andar fastal día de oy $(E E)$;

b. los judíos que lo entendieron por más, e por esto solo non usavan de comer de ninguna gordura en ninguna guisa $(G E 1)$;

c. segund que uos e contado los omnes son dios dellos. ca ellos no seyendo los fizieron \& quando se dannansabenlos adobar $(E E)$; 
d. el Rey ponia grant diligencia en todos sus feytos pero vna vez enel mes costumbraua yr a caça al mont (GCron).

En segundo lugar, la perífrasis $<$ saber + infinitivo $>$ se documenta por primera vez en iberorromance, en la obra anónima el Libro de Alexandre, que codifica la oralidad mixta, en términos de Zumthor (2001), ya que el carácter textual propio de la temática está determinado, en gran medida, por lo oral de las técnicas juglarescas con objeto de acercar lo narrado a un público ansioso por escuchar la historia de las hazañas de Alejandro Magno. Esta primera aparición ha de asociarse con lo que Jacob y Kabatek (2001: X) denominan innovaciones por expresividad, en el sentido de que el reajuste se lleva a cabo en el plano oral desde abajo, en términos de Labov (2001: 266), y ha de vincularse con la proximidad comunicativa. Prueba de ello es su total ausencia en la obra hagiográfica - inscrita en la misma corriente del Mester de Clerecía - de Gonzalo de Berceo. Obsérvese que la lectura frecuentativa del predicado sabe honrar implicaría una imagen de Dios contraria a los dogmas cristianos ya establecidos: sería impensable que el sujeto divino a quien se debe servir no siempre muestre respeto a sus fieles.

(6) A tal señor deuemos servir e aguardar/ que saue a sus sieruos de tal guisa honrar (SDom. 756 a-b).

Con todo, los pasajes del valor frecuentativo hallados en el Libro de Alexandre (7) evocan contextos moralizantes, a veces de carácter impersonal, en los que el valor de habilidad pudo solaparse fácilmente con el frecuentativo.

(7) a. al omne que non veen non saben escarnir / es bien atales omnes solitarios Bevir (LAlex, vv. 1662 c-d);

b. Los buenos e los santos que non quieren fallir / que oran, alimosnan e piensan de servir / saben con sus sermones los otros convertir / pesal tanto con estos que se quiere morir (LAlex. vv. 2400).

Su frecuencia de empleo va aumentando ligeramente a lo largo del Medievo, si bien sigue manifestando las restricciones discursivas 
vinculadas al carácter popular del giro. Según indica Yllera (1980: 203-204), hasta el siglo XV no culmina su gramaticalización (8a-c), momento a partir del cual comienza su declive.

(8) a. Ende leemos de Çesar que tanto amaua sus Caualleros commo asi mjsmo \& non sabia estar syn ellos (Castigos);

b. Los Tartres son muyt cobdiciosos et prenden volenter la cosa de otri et la suya no la saben guardar (CronCon);

c. les envió a Mateo Malferit, que sabía ser muy acepto a los seneses (Anales);

d. tenia quescrivir desta tierra a vm sola quiero dezir una y es q los hombres que saben trabajar y se dan a la virtud tienen de comer (Cartas: XVI);

e. [...] con esta galantería me inclinaba a obrar en su servicio, asegurándose observó el ser verdadero amigo de quien lo sabe ser mío y con la entereza que han experimentado Vuestras Mercedes en mis atenciones, etc. (Registro: XVII).

El descenso de su empleo en el español peninsular debe relacionarse con el triunfo de la perífrasis $<$ soler + infinitivo $>$, que, a diferencia de $<$ saber + infinitivo $>$, pierde sus restricciones distribucionales por las mismas fechas y se ve sometida a fijación, en el sentido de que el verbo principal requiere siempre infinitivo (*lo suelo). Los textos demuestran que, a medida que la expresión con soler gana frecuencia de empleo, desbanca a las otras tres construcciones perifrásticas frecuentativas, usurpando sus usos (Zieliński, 2010: 676-678), de modo que los últimos ejemplos de $<$ saber + infinitivo $>$ encontrados en la península ibérica — de carácter marcadamente popular - datan del siglo XVII (8e), aunque el valor frecuentativo de la perífrasis todavía se menciona, una centuria más tarde, en el Diccionario de autoridades (1726).

Visto todo lo anterior, el mantenimiento del valor frecuentativo de la perífrasis con saber en el español de América (sobre todo en variantes chilenas, andinas y rioplatenses) no es mera casualidad. Todo apunta a que su suerte se vio favorecida por la interferencia del quechua, 
que proporcionó un terreno abonado para los empleos populares de la perífrasis española.

(9) a. el caudal que tiene q canta su boz tiene un mulato que com pro la iglesia buena boz y no sabe andar por casa (Cartas: XVI);

b. los hombres que saben trabajar se dan a la virtud tienen de comer que los que no tantos perdidos ay en esta tier (Cartas: XVI).

Heine y Kuteva (2005: 4) insisten en que, para que opere un cambio por contacto entre dos lenguas, ha de existir algún tipo de identidad con el que los usuarios de un código lingüístico puedan identificarse fácilmente. En este caso concreto, el quechua y el español comparten una conceptualización basada en la ontología popular de que, si sabemos hacer algo, solemos hacerlo a menudo. Sin lugar a dudas, fue esta la causa que propició el auge del valor frecuentativo de la perífrasis $<$ saber + infinitivo $>$. Del mismo modo podemos explicar el valor frecuentativo que presenta la construcción en el español de Filipinas (Quilis, Casado-Fresnillo, 2008: 121-122) y su mantenimiento gracias, en este caso, al influjo del tagalo.

\section{Evolución semántica}

En esta perífrasis de carácter popular se constata la evolución semántica desde el significado de 'habilidad' al de 'frecuentatividad', ya que 'adquirir la habilidad de hacer algo' implica la lectura de 'hacerlo con frecuencia', como prueban los ejemplos ambiguos del contexto puente, en el que están presentes ambos valores. Como indicaron Bybee, Perkins y Pagliuca (1994: 15-19), el mantenimiento del significado originario no es obstáculo para que se desarrollen otros valores, como se atestigua también en los reajustes sufridos en lenguas criollas de base románica, que también han llegado al significado frecuentativo desde el de conocimiento: papiamento $s a(<$ port. saber) o haitano kôn ( $<$ fr. connaître 'conocer', 'saber') (Heine y Kuteva, 2002: 186-87).

Entra en juego, por lo tanto, el patrón de lexicalización ya mencionado previamente $(\S 1)$ (observable en el dicho español la práctica hace al maestro), con ayuda del cual el valor frecuentativo pudo 
desarrollarse en un contexto propicio. La construcción consiguió un valor más gramatical, según el planteamiento de Traugott y Dasher (2005). Factores como la negación y, ante todo, la selección de tiempos no delimitados temporalmente en indicativo favorecieron este cambio por implicatura conversacional.

Ahondando en ello, podemos afirmar que la negación juega un papel crucial en el desarrollo del valor frecuentativo, dada la premisa cultural compartida por los usuarios de un código lingüístico según la cual no saber hacer algo implica casi de inmediato no hacerlo nunca o pocas veces. Así, en el Diccionario de autoridades (1726: s.v. saber) leemos: "Se suele tomar por practicar, ò acostumbrar: y aunque tal vez se usa afirmativamente: como Fulano sabe hacer un gusto; lo mas común es con negación" (la cursiva es nuestra). De modo similar, del predicado perifrástico de (10a), no saber estar sin ellos, se infiere pragmáticamente que César no solía estar sin ellos.

Además de la negación, la elección de formas verbales no delimitadas temporalmente desempeña un papel importante. Esta restricción se debe a la incompatibilidad del valor frecuentativo con el cese de la actividad que implica el empleo de las formas verbales delimitadas temporalmente. Al codificar el carácter frecuentativo de un evento, el emisor pone de manifiesto que su realización se repite varias veces en el eje temporal, sin que le interese marcar ni su inicio ni su final. Por la misma razón la frecuentatividad resulta únicamente compatible con el presente (10a-b) y el imperfecto de indicativo (10c-d). Prueba de ello lo constituyen las formas defectivas del verbo frecuentativo por excelencia en español peninsular: soler.

(10) a. Grant honra me acreçe en tal dona tomar / la que ante quisieron con Maçeus Casar / varón que tal consejo sabe a señor dar / devrie aver vergüença ante otros fablar (LAlex. vv. $1281 \mathrm{a}-\mathrm{d}$ );

b. Solien-lo Alexe de primero llamar / mas ouo-le el padre el nombre a mudar / Paris le puso nonbre si lo oyestes / contarca igual lo fazia de los otros \& par (LAlex. vv. 360 a-d); 
c. Fueron a poca d'ora en alta mar entrados / andudieron grant tiempo radíos eerrados / eran los marineros fierament embargados / ca non sabién guiar do non eran usados (LAlex. vv. 2299 a-d);

d. Ende leemos de çesar que tanto amaua sus Caualleros commo asi mjsmo \& non sabia estar syn ellos (Castigos).

Resulta sumamente interesante comprobar en el Libro de Alexandre que el valor frecuentativo de $<$ saber + infinitivo $>$ emana únicamente de los contextos en presente de indicativo (10a), mientras que, para denotar repetición de una actividad en el pasado, el autor opta por $<$ soler + infinitivo $>$ (10c). Creemos, pues, que esto apunta a que el valor frecuentativo primero se solapó con el valor previo en contextos en presente de indicativo (llamado contexto gnómico) - recuérdese el carácter oral de la obra, con su HIC ET NUNC- Solo una vez gramaticalizada la estructura en presente, lo que sucede a partir del siglo XIV, se extiende al imperfecto.

La relativa alta frecuencia de $<$ saber + infinitivo $>$ en este contexto sintagmático provoca que el predicado compuesto empiece a adquirir un nuevo valor, pasando por una fase de ambigüedad ${ }^{2}$, como se desprende de (11a). No se sabe si las cabras son tan entendudas porque poseen la habilidad - el conocimiento adquirido - de curarse o es el emisor quien las califica de tan entendudas porque suelen curarse.

(11) a. Las cabras tan entendudas animalias son que ellas se saben melezinar $(G E$ 1);

b. E en tod esto los gascones que son yentes que non saben ueuir en paz. Passaron las montannas \& entraron en tierra de Cantabria. \& començaron de Robarla $(E E)$.

Nos encontramos, pues, ante lo que Heine (2002: 84) denomina contexto puente, es decir, una situación contextual propicia para que la expresión codifique un nuevo valor gramatical por enriquecimiento pragmático antes de convencionalizarse. Precisamente en

${ }^{2}$ Algo parecido afirma Yllera (1980: 203), para quien "la mayoría de los ejemplos $[\ldots]$ del español antiguo nos parecen dudosos puesto que pueden interpretarse más naturalmente como 'es capaz de', 'tiene poder para', 'sabe cómo' $[\ldots]$ o como "soler"'. 
esta situación contextual tan ambigua surgen dos posibles lecturas. Por una parte, es posible la antigua lectura de habilidad, que codifica creencias subjetivas del emisor sobre las actitudes adquiridas que tiene el sujeto-agente para realizar una acción, y, por otra, empieza a brotar la lectura de Aktionsart, motivada por el entorno contextual en el que ya no se focalizan las propiedades del sujeto-agente, sino el evento en sí $i^{3}$. Por lo tanto, esa lectura resulta más objetiva en comparación con la primera, dado que viene a denotar, ante todo, cómo se desarrolla la acción en el eje temporal.

\section{Conclusiones}

Como se ha demostrado en las páginas precedentes, el origen del valor frecuentativo de $<$ saber + infinitivo $>$ no es ni un calco semántico del quechua ni un cultismo, sino una expresión de raíz popular. Lo demuestra tanto su primera documentación en el Libro de Alexandre como la formación de las cuatro perífrasis frecuentativas registradas en el español medieval, en las cuales la idea de frecuencia ha llegado a codificarse como elemento rutinario de la vida cotidiana. Prueba de ello lo constituye su reajuste semántico, que parte del valor primario de habilidad. El proceso se vio favorecido por la negación y por la selección de predicados no delimitados temporalmente.

A partir del siglo XV decrece el empleo de $<$ saber + infinitivo $>$ y se incrementa el de $<$ soler + infinitivo $>$. Dos centurias más tarde, cae en desuso en el español peninsular, pero se conserva en el de zonas del sur de América por influencia del quechua, donde desbanca a otras construcciones de la misma índole, convirtiéndose en el español rioplatense en la única perífrasis de frecuentatividad. Asimismo, se mantuvo en el español de Filipinas por influjo del tagalo.

3 "Si la lectura del primer significado resulta más subjetiva, porque parte de las creencias del emisor, la segunda, en cambio, adquiere una perspectiva más objetiva en contraste con la primera, dado que ya no se basa en creencias, sino en su percepción, que, pese a su innegable e incuestionable parcialidad, arranca de un ángulo más objetivo que puede ser compartido por el destinatario" (Zieliński, 2014: 133). 


\section{Relación de fuentes}

Anales = Jerónimo Zurita (1562/1967), Anales de la corona de Aragón, ed. de Á. Canellas López, CSIC, Zaragoza.

BREPOLIS = Brepols Publishers, Library of Latin Texts, [on-line] http:// www.brepolis.net, 12.2016.

Cartas $=$ VV.AA. (s. XVI/2009), Cartas de particulares en Indias del siglo XVI, ed. de M. Fernández Alcaide, Iberoamericana Vervuert, Madrid-Frankfurt am Main.

Castigos = Anónimo (1293/1952), Castigos e documentos para bien vivir ordenados por el rey Sancho IV, ed. A. Rey, Indiana University Publications, Bloomington.

CORDE = Real Academia Española, Corpus diacrónico del español, [on-line] http://www.rae.es, 12.2016.

CORDIAM =Academia Mexicana de la Lengua, Corpus Diacrónico y Diatópico del Español de América (cordiam), [on-line] www.cordiam.org, 12.2016 .

$\boldsymbol{C R E A}=$ Real Academia Española, Corpus de referencia del español actual, [on-line] http://www.rae.es, 12.2016.

CronCon $=$ Fernández de Heredia, J. (h. 1350), Crónica de los conqueridores I y II, ADMYTE.

$\boldsymbol{E} \boldsymbol{E}=$ Alfonso X el Sabio (1270/2002), Estoria de Espanna que fizo el muy noble rey don Alfonsso, fijo del rey don Fernando et de la reyna donna Beatriz [edición paleográfica], ed. de P. Sánchez-Prieto Borja, Universidad de Alcalá de Henares, Alcalá de Henares.

GCron = Fernández de Heredia, J. (h. 1376/2003), Gran Crónica de España, ed. de J. M. Cacho Blecua, Universidad de Zaragoza, Zaragoza.

GE.1 = Alfonso X el Sabio (1275/2002), Estoria General. Primera parte [edición digital paleográfica], ed. de P. Sánchez-Prieto Borja, Universidad de Alcalá de Henares, Alcalá de Henares.

LAlex $=($ h.1250/1906), Libro de Alexandre, ed. de A. Morel Fatio, Max Niemeyer, Dresden.

SDom $=$ Gonzalo de Berceo (h. 1236/1972), Vida de Santo Domingo de Silos, ed. de T. Labarta de Chaves, Castalia, Madrid. 


\section{Bibliografía}

BYBEE, J. L., PERKINS, R. D., PAGLIUCA, W. (1994), The Evolution of Grammar. Tense, Aspect, and Modality in the Languages of the World, University of Chicago Press, Chicago.

ENRIQUE-ARIAS, A. (dir.) (2008), Biblia Medieval, [on-line], http://www. bibliamedieval.es.

ERNOUT, A., MEILLET, A. (1951), Dictionnaire étymologique de la langue latine: histoire des mots, 3 vols, Klincksieck, Paris.

FERNÁNDEZ DE CASTRO, F. (1999), Las perifrasis verbales en el español actual, Gredos, Madrid.

GARCÍA FERNÁNDEZ, L. (dir.) (2006), Diccionario de perífrasis verbales, Gredos, Madrid.

HEINE, B., KUTEVA, T. (2002), World Lexicon of Grammaticalization, Cambridge University Press, Cambridge, [on-line] https://doi. org/10.1017/CBO9780511613463.

HEINE, B., KUTEVA, T. (2005), Language Contact and Grammatical Change, Cambridge University Press, Cambridge, [on-line] https://doi. org/10.1017/CBO9780511614132.

JACOB, D., KABATEK, J. (2001), "Introducción: Lengua, texto y cambio lingüístico en la Edad Media iberorrománica”, en: Jacob, D., Kabatek, J. (eds.), Lengua medieval y tradiciones discursivas en la Península Ibérica. Descripción gramatical - pragmática histórica - metodología, Iberoamericana Vervuert, Madrid-Frankfurt am Main, pp. VII-XVIII.

KANY, Ch. E. (1994), Sintaxis hispanoamericana, Gredos, Madrid.

LABOV, W. (2001), Principles of Language Change, vol. 2: Social Factors. Language in Society, Blackwell Publishers, Oxford.

LEWIS, Ch. T., SHORT, Ch. (1969), A Latin Dictionary. Founded on Andrews' Edition of Freund's Latin Dictionary, Oxford at the Clarendon Press, Oxford.

LIDA DE MALKIEL, M. R. (1948), “«Saber» «soler» en las lenguas romances y sus antecedentes grecolatinos", Romance Philology, 2, pp. 269-283.

OLBERTZ, H. (1998), Verbal Periphrases in a Functional Grammar of Spanish, Mouton de Gruyter, Berlin-New York, [on-line] https://doi. org/10.1515/9783110820881. 
QUILIS, A., CASADO-FRESNILLO, C. (2001), La lengua española en Filipinas, CSIC, Madrid.

Real Academia Española (1726), Diccionario de Autoridades, [on-line] http:// buscon.rae.es/ntlle/SrvltGUILoginNtlle, 12.2016.

Real Academia Española y Asociación de Academias de la Lengua Española (2009), Nueva gramática de la lengua española, 2 vols., Espasa, Madrid.

TALMY, L. (1985), "Lexicalization Patterns: Semantic Structure in Lexical Forms", en: Shopen, T. (ed.), Language Typology and Syntactic Description, vol. 3: Grammatical Categories and the Lexicon, Cambridge University Press, Cambridge, pp. 57-149.

TRAUGOTT, E. C., DASCHER, R. (2001), Regularity in Semantic Change, Cambridge University Press, Cambridge, [on-line] https://doi. org/10.1017/CBO9780511486500.

VÁZQUEZ, H. (1980), El quichua en nuestro lenguaje popular, Departamento de Difusión Cultural de la Universidad de Cuenca, Cuenca.

VENDLER, Z. (1957), "Verbs and Times", The Philosophical Review 66(2), pp. 143-160.

YLLERA, A. (1980), Sintaxis histórica del verbo español. Las perifrasis medievales, Departamento de Filología Francesa. Universidad de Zaragoza, Zaragoza.

ZAMORA, V. (1974), Dialectología española, Gredos, Madrid.

ZIELIŃSKI, A. (2010), "Las perífrasis frecuentativas. Su origen y gramaticalización en español medieval y clásico", en: Górnikiewicz, J., Grzmil-Tylutki, H., Piechnik, I. (eds.), En quête de sens. Études dédiées à Marcela Świątkowska, Wydawnictwo Uniwersytetu Jagiellońskiego, Kraków, pp. 674-682.

ZIELIŃSKI, A. (2014), Perífrasis de los verbos de movimiento en español medieval y clásico, Wydawnictwo Uniwersytetu Jagiellońskiego, Kraków.

ZUMTHOR, P. (2001), A letra e a voz. A literatura medieval, Compahnia Das Letras, São Paulo. 